\title{
Test of universal scaling of ac conductivity in ionic conductors
}

\author{
C. León \\ GFMC, Dpto. Física Aplicada III, Facultad de Ciencias Físicas, Universidad Complutense de Madrid, Avda. Complutense s/n, \\ 28040 Madrid, Spain \\ P. Lunkenheimer \\ Experimentalphysik V, Universität Augsburg, D-86135, Augsburg, Germany \\ K. L. Ngai \\ Naval Research Laboratory, Washington, DC 20375-5320 \\ (Received 13 January 2000; revised manuscript received 26 July 2001; published 18 October 2001)
}

\begin{abstract}
Electrical relaxation data of crystalline yttria-stabilized zirconia are used to analyze the permittivity change observed in the spectra of the real part of the permittivity in ionic conducting materials. It is found that this permittivity change is independent of both temperature and mobile-ion concentration, and it is determined solely by the degree of interaction among ions in the relaxation process. This finding is at odds with an expression for the permittivity change in the framework of a proposed universal ac conductivity scaling law for glassy ionic conductors. On the other hand, not only the total permitivity change, but also the particular frequency dependence of the permittivity spectra is found to be consistent with the analysis of electrical relaxation in terms of the electric modulus. The results of this work give further support to the use of the electric modulus in describing electrical relaxation in ionic conductors.
\end{abstract}

DOI: 10.1103/PhysRevB.64.184304

PACS number(s): 66.10.Ed, 66.30.Dn, 66.30.Hs

\section{INTRODUCTION}

Electrical relaxation measurements are commonly used to characterize the dynamics of ionic transport in ionically conducting materials. ${ }^{1-25}$ Most of these measurements are performed in the frequency domain, and the experimental information obtained is often presented and analyzed in terms of the complex conductivity, $\sigma^{*}(\omega)=\sigma^{\prime}(\omega)+i \sigma^{\prime \prime}(\omega)$. Alternative representations of the same experimental data are the complex permittivity $\varepsilon^{*}(\omega)=\varepsilon^{\prime}(\omega)-i \varepsilon^{\prime \prime}(\omega)$ and the complex electric modulus $M^{*}(\omega)=M^{\prime}(\omega)+i M^{\prime \prime}(\omega)$, which are related to the complex conductivity by

$$
\varepsilon^{*}(\omega)=\frac{\sigma^{*}(\omega)}{i \omega \varepsilon_{0}}
$$

and

$$
M^{*}(\omega)=\frac{1}{\varepsilon^{*}(\omega)}=\frac{i \omega \varepsilon_{0}}{\sigma^{*}(\omega)},
$$

where $\varepsilon_{0}$ is the permittivity of the free space.

It is clear that, whichever representation is used, one is considering the same experimental data, and therefore, there should be no difference in the results and conclusions derived from the different approaches. However, in reality different approaches have led some workers to different conclusions. Perhaps the most dramatic difference of the different approaches is the interpretation of the dependence of the electrical relaxation dispersion on the ionic concentration. Workers using the electric modulus representation ${ }^{7,8,10,19,20}$ have found that the dispersion narrows towards a single exponential as ionic concentration is decreased, suggesting that the width of the dispersion comes from ion-ion interaction as proposed by the coupling model..$^{9,18,25,26}$

A totally different view is offered by some workers who use the real part of the complex conductivity, $\sigma^{\prime}(\omega)$, to examine data. It has been recently proposed that data sets of the real part of the complex conductivity, $\sigma^{\prime}(\omega)$, of various glassy ionic conductors containing a wide range of ion concentrations and at different temperatures can be scaled into a single master curve. ${ }^{21-23}$ Roling et al. ${ }^{21,22}$ showed that the scaling law

$$
\sigma^{\prime}(\omega) / \sigma_{d c}=F\left(\frac{x}{\sigma_{d c} T} \omega\right)
$$

holds in a series of alkali borate glasses $\left(\mathrm{Na}_{2} \mathrm{O}\right)_{x}\left(\mathrm{~B}_{2} \mathrm{O}_{3}\right)_{1-x}$ with different mobile-ion concentrations in the relatively small range $0.1<x<0.3$. Sidebottom ${ }^{23}$ analyzed another glass system $\left(\mathrm{Na}_{2} \mathrm{O}\right)_{x}\left(\mathrm{GeO}_{2}\right)_{1-x}$ over a much wider range of sodium ion concentration $(0.003<x<0.1)$ using the scaling law proposed by Roling et al., and he found that it fails to collapse all conductivity data onto a common curve. He proposed another scaling law of the form

$$
\sigma^{\prime}(\omega) / \sigma_{d c}=F\left(\frac{\varepsilon_{0} \Delta \varepsilon}{\sigma_{d c}} \omega\right),
$$

which involves the permittivity change

$$
\Delta \varepsilon=\varepsilon_{s}-\varepsilon_{\infty} .
$$

In Eq. (5), $\varepsilon_{\infty}$ is the high-frequency permittivity value determined from $\varepsilon^{\prime}(\omega)$ at sufficiently high frequencies, and $\varepsilon_{s}$ is the low-frequency value contributed from mobile ions, which is difficult to obtain from experimental data due to the usual presence of electrode polarization effects at low fre- 
quencies. According to Sidebottom, the hop of the mobile ion between sites is analogous to the rotation of a permanent dipole. From this analogy, he proposed that the Debye model for dipolar relaxation should give a suitable approximation to the permittivity change

$$
\Delta \varepsilon=\frac{\gamma N(q d)^{2}}{3 \varepsilon_{0} k T},
$$

where $N$ is the total mobile-ion concentration, $\gamma$ is the fraction of $N$ which are effectively mobile, $q$ is the charge of the mobile ions, $d$ is the distance traversed in a single hop, and the product $q d$ is the effective dipole of the hopping ion. A similar expression for $\Delta \varepsilon$ has been suggested by Roling. ${ }^{22}$

In both scaling procedures suggested for the real part of the complex conductivity, the ion dynamics is described by the same master function $F(u)$ in Eqs. (3) and (4), which follows approximately a power-law frequency dependence at high frequencies of the form $F(u) \approx 1+u^{n}$, with an exponent $n \approx 0.65$. $^{23}$ The results have been interpreted by the proponents of scaling laws as evidence of the existence of a universal relaxation process in ionic conductors, which is independent of temperature and the effective mobile ion density. Although, up to now, there has been no theory or model proposed to explain why such $F(u)$ governs the frequency dispersion of many ionic conductors. Since the same data in the electric modulus representation do not scale to any master curve, proponents of the universal scaling of the conductivity have no choice but to discredit the electric modulus representation of data. They went on to say that the conductivity, instead of the electric modulus, is the quantity more directly related to the microscopic dynamics of the ions and is the appropriate one to analyze electrical relaxation of ionically conducting materials. However, a recently published work has shown otherwise, namely, that the electric modulus spectrum is a mirror image of the microscopic ion-hopping spectrum. The only difference is a shift from the macroscopic frequency of the former to the microscopic frequency of the latter. ${ }^{27}$

Conclusions drawn by the proponents of scaling laws, if true, would have a large impact on the interpretation of the electrical relaxation data of ionic conductors and, in particular, the interpretation of the permittivity change $\Delta \varepsilon$. Those authors defending the use of the electric modulus in describing the ionic transport dynamics have maintained a different interpretation of $\Delta \varepsilon$ for a long time. ${ }^{1-4,6,7,19,24,27,28}$ According to them, the permittivity change $\Delta \varepsilon=K \varepsilon_{\infty}$ is a product of the high-frequency permittivity value and a function $K$ which is solely determined by the shape of the electric modulus spectrum. This is very different from the expression given by Eq. (6) for $\Delta \varepsilon$, which depends on temperature, mobile-ion density, and the ion-hopping distance. Thus, these drastically different interpretations of $\Delta \varepsilon$ underscore the severe disparity in the theoretical interpretations of electrical relaxation.

It must be mentioned that all ionic conducting materials used in the previously proposed conductivity scaling are glasses or melts and therefore the ionic hopping distance $d$ is not known or well defined in these materials. An estimate of this quantity can be obtained by using Eq. (6), but there is no way to decide definitely about the validity of this expression and the interpretation given for the proposed scaling laws. On the other hand, electrical relaxation measurements of a crystalline ionic conductor, in which the mobile ion density $N$ and the ionic-hopping distance $d$ are known, would constitute an ideal and critical test of the validity of Eq. (6) and the alternative interpretation of the permittivity change $\Delta \varepsilon$.

In this paper we present electrical relaxation data of a crystalline ionic conductor at different temperatures and for samples with different mobile ion densities, and show that the observed permittivity change $\Delta \varepsilon$ is both temperature and composition independent. Moreover, not only the value of $\Delta \varepsilon$, but the particular frequency dependence observed in $\varepsilon^{\prime}(\omega)$ from $\varepsilon_{\infty}$ to $\varepsilon_{s}$ is found to be consistent with the analysis of electrical relaxation in terms of the electric modulus.

\section{ELECTRICAL RELAXATION IN YTTRIA-STABILIZED ZIRCONIA}

We have chosen $\left(\mathrm{ZrO}_{2}\right)_{1-x}\left(\mathrm{Y}_{2} \mathrm{O}_{3}\right)_{x}$, yttria-stabilized zirconia (YSZ), as an ideal material to test the validity of Eq. (6). YSZ is a crystalline ionic conductor with a cubic fluorite structure. Doping zirconia with trivalent yttria atoms creates oxygen vacancies in the crystal structure, and results in a high ionic conductivity due to the hopping of oxygen anions through the oxygen vacancies in the lattice. ${ }^{15,16}$ Conductivity relaxation data of samples of $8 \%, 10 \%$, and $12 \%$ molar yttria content have been analyzed, in the frequency range $20 \mathrm{~Hz}$ to $1 \mathrm{MHz}$ and at temperatures between 300 and $800 \mathrm{~K}$. The 8 mol \% $\mathrm{Y}_{2} \mathrm{O}_{3}$ sample was obtained from Kerafol Co., and single crystals with $10 \mathrm{~mol} \%$ and $12 \mathrm{~mol} \% \mathrm{Y}_{2} \mathrm{O}_{3}$ were grown from the melt under standard atmospheric conditions. ${ }^{16}$ Further experimental details are given in Ref. 16 , where some of the present data were already evaluated within both electric modulus and conductivity representations.

The frequency dependence of the real part of the permittivity, $\varepsilon^{\prime}(\omega)$, is shown in Fig. 1 for samples with different yttria content and at several temperatures. Data points have been shifted horizontally in a log-log scale by using a normalization frequency for each temperature, $f_{p}$, which was determined in order to collapse all data on a single curve. In spite of the emergence of strong electrode polarization effects at high temperature and low frequencies, a clear plateau is present at moderately low frequencies, and the permittivity change from the high-frequency permittivity $\varepsilon_{\infty}$ towards a low-frequency value of $\varepsilon_{s}$ can be determined. It is found that $\varepsilon_{\infty}=28 \pm 1$ in the whole temperature and composition ranges considered.

We can also see by inspection of Fig. 1 that the magnitude of $\Delta \varepsilon$ seems to be nearly independent of temperature and also of the mobile-ion density. The observed insensitivity of $\varepsilon_{s}$ or $\Delta \varepsilon$ to temperature is in serious disagreement with a change of $\varepsilon_{s}$ by a factor of about 1.6 predicted by Eq. (6) in the temperature range of 500-780 K, where the value of $\varepsilon_{s}$ can be determined from the experimental data. This suggests that this permittivity change $\Delta \varepsilon$ might be related to the degree of ion-ion interaction and correlation in the electrical relaxation process, as suggested by the coupling model inter- 
pretation of the data in the electric modulus representation, instead of being due to an effective dipole moment $(q d)$ arising from single-ion hopping as described by Eq. (6).

\section{ELECTRIC MODULUS ANALYSIS AND CALCULATION OF $\varepsilon(\omega)$ AND $\varepsilon_{s}$}

The electric modulus formalism ${ }^{1-4}$ for the analysis of experimental macroscopic dielectric data starts with the argument that for mobile ions the appropriate quantity to consider is the decay of the electric field, $E(t)$, under the constraint of a constant displacement vector $D(t)$ :

$$
E(t)=E(0) \Phi(t),
$$

where $E(0)$ is the initial electric field and $\Phi(t)$ is the relaxation function. In the frequency domain, the electrical relaxation is appropriately represented by the electric modulus

$$
M^{*}(\omega)=\frac{1}{\varepsilon_{\infty}}\left[1-\int_{0}^{\infty} e^{-i \omega t}\left(-\frac{d \Phi}{d t}\right) d t\right] .
$$

In general, the high-frequency and low-frequency limits of the real part of the permittivity, $\varepsilon_{\infty}$ and $\varepsilon_{s}$, are connected by ${ }^{1-4}$

$$
\varepsilon_{s}=\varepsilon_{\infty}\left\langle\tau^{2}\right\rangle /\langle\tau\rangle^{2}
$$

where

$$
\left\langle\tau^{n}\right\rangle=\int_{0}^{\infty} t^{n-1} \Phi(t) d t
$$

Of the well-behaved, one-parameter relaxation functions, the one which gives the best fit to typical experimental data is the stretched exponential function, also known as the Kohlrausch-Williams-Watts (KWW) function, ${ }^{29}$

$$
\Phi(t)=\exp \left[-(t / \tau)^{\beta}\right]
$$

The exponent $\beta$ is a fractional number, which typically ${ }^{26}$ falls in the range $0.4<\beta<0.7$ except when the ion concentration is very low. The quantity $(1-\beta)$ is a measure of the degree of correlation between ions in the ionic transport. The limit of $(1-\beta)$ going to zero corresponds to completely uncorrelated motion of mobile ions expected at extremely low ion concentrations. For this relaxation function, it can be shown by straightforward mathematics ${ }^{1-4,7,24}$ that the value of the permittivity in the limit of low frequencies, $\varepsilon_{s}$, is given by

$$
\varepsilon_{s}=\beta \frac{\Gamma(2 / \beta)}{[\Gamma(1 / \beta)]^{2}} \varepsilon_{\infty},
$$

where $\Gamma$ is the gamma function. Therefore, the permittivity change $\Delta \varepsilon$ caused by the relaxation of hopping ions is given by $^{24}$

$$
\Delta \varepsilon=\left[\beta \frac{\Gamma(2 / \beta)}{[\Gamma(1 / \beta)]^{2}}-1\right] \varepsilon_{\infty} .
$$
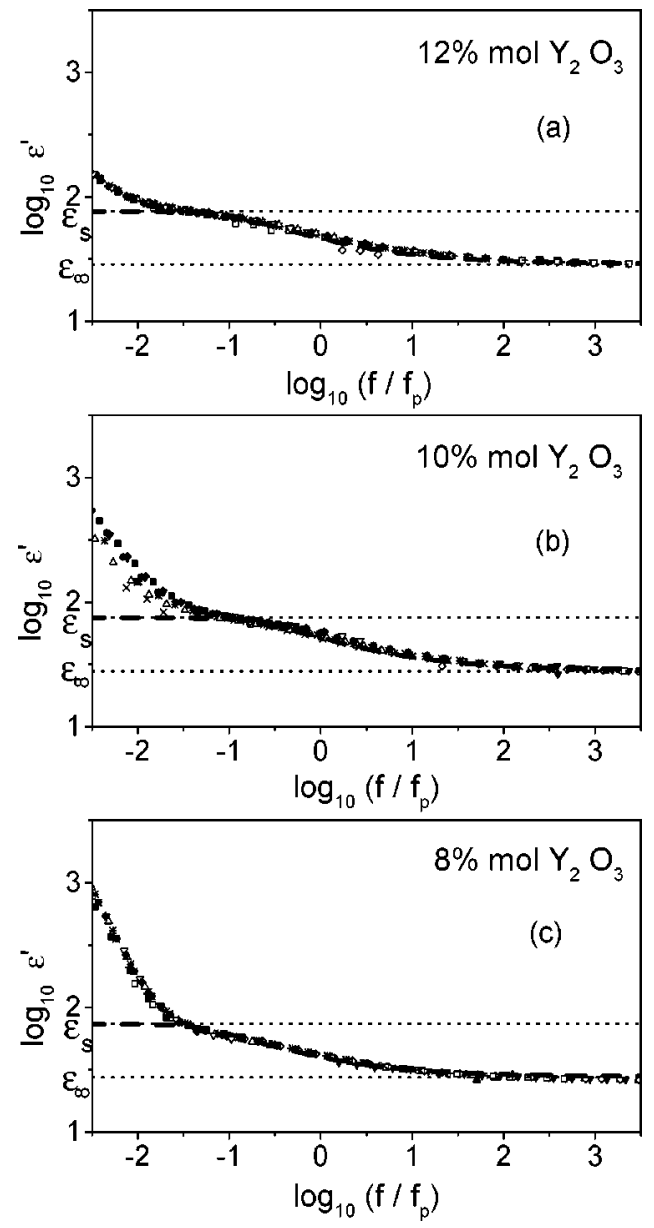

FIG. 1. Log-log plots of the real permittivity vs frequency for (a) $12 \mathrm{~mol} \% \mathrm{Y}_{2} \mathrm{O}_{3}-\mathrm{YSZ}$ at $302 \mathrm{~K}(\mathrm{O}), 343 \mathrm{~K}(\boldsymbol{\Delta}), 383 \mathrm{~K}(\boldsymbol{\nabla})$, $423 \mathrm{~K}(\diamond), 464 \mathrm{~K}(\square), 503 \mathrm{~K}(+), 544 \mathrm{~K}(\times), 583 \mathrm{~K}(*), 624$ $\mathrm{K}(\bullet), 664 \mathrm{~K}(\triangle), 704 \mathrm{~K}(\nabla), 744 \mathrm{~K}(\bullet)$, and $774 \mathrm{~K}(\boldsymbol{\square})$; (b) $10 \mathrm{~mol} \% \mathrm{Y}_{2} \mathrm{O}_{3}-\mathrm{YSZ}$ at $300 \mathrm{~K}(\mathrm{O}), 340 \mathrm{~K}(\boldsymbol{\Delta}), 380 \mathrm{~K}(\boldsymbol{\nabla}), 420$ $\mathrm{K}(\diamond), 460 \mathrm{~K}(+), 500 \mathrm{~K}(\square), 540 \mathrm{~K}(\times), 580 \mathrm{~K}(*), 620 \mathrm{~K}$ $(\bullet), 660 \mathrm{~K}(\triangle), 700 \mathrm{~K}(\nabla), 740 \mathrm{~K}(\diamond)$, and $775 \mathrm{~K}(\mathbf{\square})$; (c) 8 $\mathrm{mol} \% \mathrm{Y}_{2} \mathrm{O}_{3}-\mathrm{YSZ}$ at $300 \mathrm{~K}(\mathrm{O}), 340 \mathrm{~K}(\boldsymbol{\Delta}), 380 \mathrm{~K}(\boldsymbol{\nabla}), 420 \mathrm{~K}$ $(\diamond), 460 \mathrm{~K}(\square), 500 \mathrm{~K}(+), 540 \mathrm{~K}(\times), 580 \mathrm{~K}(*), 620 \mathrm{~K}(\bullet)$, $660 \mathrm{~K}(\triangle), 700 \mathrm{~K}(\nabla), 740 \mathrm{~K}(\diamond)$, and $780 \mathrm{~K}(\boldsymbol{\square})$. The frequency axis has been shifted for each data set in order to collapse the permittivity data at different temperatures onto a single curve. The normalization frequencies $f_{p}$ used for each sample were calculated from the following expressions: $\log f_{p}=9.81-5577 / T$ in (a), $\log f_{p}=11.13-5052 / \mathrm{T}$ in (b), and $\log f_{p}=11.75-4882 / \mathrm{T}$ in (c). Dashed lines represent the real part of the permittivity, $\varepsilon^{\prime}(\omega)$, calculated from the fits of the electric modulus data by a KWW function with $\beta=0.52$ (see text). Horizontal dotted lines represent the limiting values $\varepsilon_{\infty}$ and $\varepsilon_{s}$ at high and low frequencies, respectively. The permittivity change $\Delta \varepsilon=\varepsilon_{s}-\varepsilon_{\infty}$ is found to be independent of both temperature and mobile ion concentration.

It is worthwhile to emphasize that the proportionality relationship between $\Delta \varepsilon$ and $\varepsilon_{\infty}$ holds for any choice of $\Phi(t)$ to describe electrical relaxation. Equation (13) is obtained for the choice of a KWW function, but a similar expression should be obtained for any other choice, and a comparable value of the proportionality factor should be obtained from the corresponding expression of the parameter (or param- 
eters) describing the nonexponential time dependence of the relaxation function instead of the stretch parameter $\beta$. More importantly, like the fractional value of $(1-\beta)$, the magnitude of $\Delta \varepsilon$ depends on the degree of ion-ion correlations [see Eq. (13)]. The magnitude of $\Delta \varepsilon$ would be zero for the ideal case where ion-ion correlations are nonexistent. The proportionality factor in Eq. (13) and hence $\Delta \varepsilon$ increase as (1 $-\beta$ ) increases when the ion-ion correlations become more important. This is in contrast with the expression for $\Delta \varepsilon$ given by Eq. (6), which indicates that $\Delta \varepsilon$ depends neither on $\varepsilon_{\infty}$ nor on any quantity related to the correlation between the ions in transport.

In order to compare the permittivity change $\Delta \varepsilon$ observed in our samples with that predicted by Eq. (13) we have analyzed experimental data in terms of the electric modulus approach and determine the best fit of the relaxation function $\Phi(t)$ to a stretched exponential function. We have found that electrical relaxation data are well described by using a single exponent $\beta$, independent of both temperature and yttria content in the experimentally available narrow range of $8 \%-$ $12 \%$. In Fig. 2 we present the real and imaginary parts of the electric modulus spectra of samples with $12 \%$ and $8 \%$ yttria contents at several temperatures. Lines in the figure are fits of experimental data using in all cases a stretched exponential with $\beta=0.52$ for the relaxation function $\Phi(t)$. This finding, together with the fact that $\varepsilon_{\infty}$ is almost constant, implies that the permittivity change $\Delta \varepsilon$ is also approximately constant according to Eq. (13). Therefore $\Delta \varepsilon$ is independent of temperature and composition and its predicted value from the electric modulus analysis is $\Delta \varepsilon=1.75 \varepsilon_{\infty}=49 \pm 2$.

On the other hand, according to Eq. (6), the permittivity change should depend on temperature and mobile ion density. It is known from the crystal structure of YSZ that the hopping distance of oxygen ions is $2.57 \AA$. For the yttria concentrations used in this work, the mobile-ion density (oxygen vacancies) varies between $2.18 \times 10^{27} \mathrm{~m}^{-3}$ and 3.16 $\times 10^{27} \mathrm{~m}^{-3}$ for samples with $8 \%$ and $12 \%$ molar yttria content, respectively. Assuming that all the oxygen vacancies contribute to the oxygen motion $(\gamma=1)$, the permittivity change should vary between $\Delta \varepsilon=70$, for the sample with $8 \%$ yttria at $580 \mathrm{~K}$, and $\Delta \varepsilon=138$, for the sample with $12 \%$ yttria at $423 \mathrm{~K}$. The calculated permittivity change is significantly larger than observed, and its temperature and composition dependences also contradict the experimental data.

Dashed lines in Figs. 1(a)-1(c) represent the real part of the permitivitty, $\varepsilon^{\prime}(\omega)$ calculated [Eq. (2)] from the fits of the electric modulus data by the stretched exponential function with $\beta=0.52$ [Eqs. (8) and (11)]. From these fits the values of $\varepsilon_{\infty}=28 \pm 1$ and $\varepsilon_{s}=77 \pm 2$ were obtained at high and low frequencies, respectively. It can be seen that there is excellent agreement of the calculated $\varepsilon^{\prime}(\omega)$ with experimental data, not only in the magnitude of the permittivity change, but also in the frequency dependence. This strongly suggests the validity of Eq. (13) instead of Eq. (6) to account for $\Delta \varepsilon$ and that the electric modulus is the appropriate approach to describe electrical relaxation in ionic conductors.

\section{CONCLUSION}

From electrical relaxation data of yttria-stabilized zirconia, a crystalline ionic conductor, we have shown that the
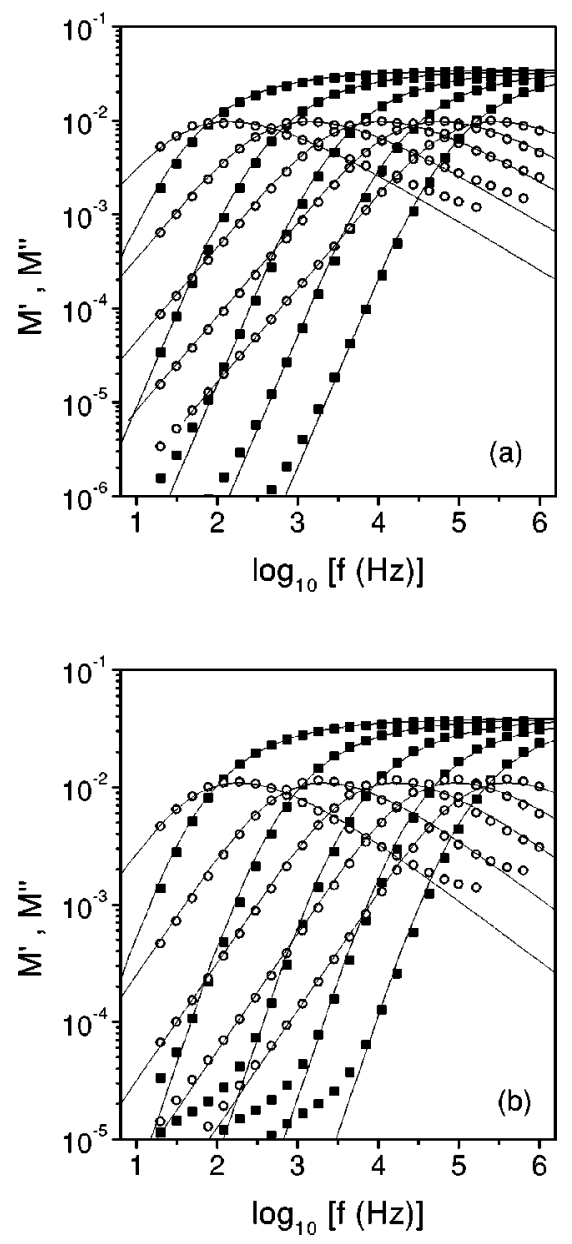

FIG. 2. Log-log plots of the real (solid squares) and imaginary (open circles) parts of the electric modulus vs frequency for YSZ samples with different yttria content and at different temperatures, increasing temperature from left to right: (a) $12 \mathrm{~mol} \% \mathrm{Y}_{2} \mathrm{O}_{3}-\mathrm{YSZ}$ at $464 \mathrm{~K}, 503 \mathrm{~K}, 544 \mathrm{~K}, 583 \mathrm{~K}$, and $624 \mathrm{~K}$; (b) $8 \mathrm{~mol} \% \mathrm{Y}_{2} \mathrm{O}_{3}-\mathrm{YSZ}$ at $420 \mathrm{~K}, 460 \mathrm{~K}, 500 \mathrm{~K}, 540 \mathrm{~K}$, and $580 \mathrm{~K}$. Solid lines are KWW fits to electric modulus data and $\beta=0.52$ in all cases.

frequency dependence of $\varepsilon^{\prime}(\omega)$ including the observed magnitude of the permittivity change $\Delta \varepsilon$ is in excellent quantitative agreement with that calculated from the stretched exponential fit to the data in the electric modulus representation. In particular, as obtained from Eq. (13), $\Delta \varepsilon$ is related solely to the high-frequency permittivity value $\varepsilon_{\infty}$ and to the nonexponential character of the relaxation function $\Phi(\mathrm{t})$, which is determined by the degree of interaction and correlation among ions in the ion-hopping relaxation process. The value of the parameter $\beta$ obtained in the stretched exponential fits is also a measure of the nonexponential character of the relaxation. We have found nearly constancy of $\beta, \varepsilon_{\infty}$, and $\Delta \varepsilon$ with change in temperature and ion concentration, contrary to that expected from Eq. (6).

We have also checked our experimental data against Eq. (6), recently proposed from the scaling of the real part of the ac conductivity in ionic conducting materials. Equation (6) predicts a large variation of $\Delta \varepsilon$ within the temperature and composition range investigated, at variance with the observed near constancy of the permittivity change. The anal- 
ogy of a hop of a mobile ion between sites to the rotation of a permanent dipole used to justify Eq. (6) should be applicable to crystalline as well as glassy or molten ionic conductors. In view of this failure and in order to salvage the situation, one might have to resort to the argument that the proposed equation (6) is applicable to glassy or molten ionic conductors and not to crystalline ionic conductors. Nonetheless, the conductivity relaxation data of crystalline ionic conductors $^{13,15,16,30,31}$ exhibit the same characteristics of glassy and molten ionic conductors. Thus, at least from a phenomenological point of view the conductivity relaxation mechanism is the same and whatever theory or model proposed for one should apply to the other. Certainly, the con- ductivity relaxation controlled by the ion-ion interaction and correlation being present in crystalline, glassy, and molten ionic conductors is consistent with the generality of the characteristics. It is important that any other proposed mechanism should also do the same.

\section{ACKNOWLEDGMENTS}

The work performed at the Naval Research Laboratory was supported by ONR. We thank J. Ullrich for help in the dielectric measurements and J. Santamaría and C. T. Moynihan for helpful discussions.
${ }^{1}$ P.B. Macedo, C.T. Moynihan, and R. Bose, Phys. Chem. Glasses 13, 171 (1972).

${ }^{2}$ V. Provenzano, L.P. Boesch, V. Volterra, C.T. Moynihan, and P.B. Macedo, J. Am. Ceram. Soc. 55, 492 (1972).

${ }^{3}$ C.T. Moynihan, L.P. Boesch, and N.L. Laberge, Phys. Chem. Glasses 14, 122 (1973).

${ }^{4}$ F.S. Howell, R.A. Bose, P.B. Macedo, and C.T. Moynihan, J. Phys. Chem. 78, 639 (1974).

${ }^{5}$ A.K. Jonscher, Dielectric Relaxation in Solids (Chelsea Dielectric Press, London, 1983).

${ }^{6}$ C.A. Angell, Chem. Rev. 90, 523 (1990).

${ }^{7}$ J.H. Simmons, P.B. Elterman, C.J. Simmons, and R.K. Mohr, J. Am. Ceram. Soc. 62, 158 (1979).

${ }^{8}$ J.F. Cordaro and M. Tomozawa, J. Am. Ceram. Soc. 64, 713 (1981)

${ }^{9}$ K.L. Ngai, R.W. Rendell, and H. Jain, Phys. Rev. B 30, 2133 (1984).

${ }^{10}$ H.K. Patel and S.W. Martin, Phys. Rev. B 45, 10292 (1992).

${ }^{11}$ C. Cramer, K. Funke, and T. Saatkamp, Philos. Mag. B 71, 701 (1995).

${ }^{12}$ D.L. Sidebottom, P.F. Green, and R.K. Brow, Phys. Rev. B 56, 170 (1997).

${ }^{13}$ C. León, J. Santamaría, M.A. París, J. Sanz, J. Ibarra, and L.M. Torres, Phys. Rev. B 56, 5302 (1997).

${ }^{14}$ P. Lunkenheimer, A. Pimenov, and A. Loidl, Phys. Rev. Lett. 78, 2995 (1997).
${ }^{15}$ C. León, M.L. Lucía, and J. Santamaría, Phys. Rev. B 55, 882 (1997).

${ }^{16}$ A. Pimenov, J. Ullrich, P. Lunkenheimer, A. Loidl, and C.H. Rüscher, Solid State Ionics 109, 111 (1998).

${ }^{17}$ C. Cramer and M. Buscher, Solid State Ionics 105, 109 (1998).

${ }^{18}$ K.L. Ngai, J. Non-Cryst. Solids 248, 194 (1999).

${ }^{19}$ K.L. Ngai and C.T. Moynihan, MRS Bull. 23 (11), 51 (1998).

${ }^{20}$ H. Jain and S. Krishnaswami, Solid State Ionics 105, 129 (1998).

${ }^{21}$ B. Roling, A. Happe, K. Funke, and M.D. Ingram, Phys. Rev. Lett. 78, 2160 (1997).

${ }^{22}$ B. Roling, Solid State Ionics 105, 185 (1998).

${ }^{23}$ D.L. Sidebottom, Phys. Rev. Lett. 82, 3653 (1999).

${ }^{24}$ K.L. Ngai and R.W. Rendell, Phys. Rev. B 61, 9393 (2000).

${ }^{25}$ K.L. Ngai, Phys. Rev. B 48, 13481 (1993); J. Chem. Phys. 98, 6424 (1993).

${ }^{26}$ K.L. Ngai, G.N. Greaves, and C.T. Moynihan, Phys. Rev. Lett. 80, 1018 (1998).

${ }^{27}$ K.L. Ngai and C. León, Phys. Rev. B 60, 9396 (1999).

${ }^{28}$ H. Wagner and R. Richter, J. Appl. Phys. 85, 1750 (1999).

${ }^{29}$ R. Kohlrausch, Ann. Phys. (Leipzig) 72, 393 (1847); G. Williams and D.C. Watts, Trans. Faraday Soc. 66, 80 (1970).

${ }^{30}$ K.L. Ngai and U. Strom, Phys. Rev. B 38, 10350 (1988).

${ }^{31}$ B. Munro, M. Schrader, and P. Heitjans, Ber. Bunsunger, Ber. Bunsenges. Phys. Chem. 96, 1718 (1992); W. Franke and P. Heitjans, ibid. 96, 1674 (1992). 